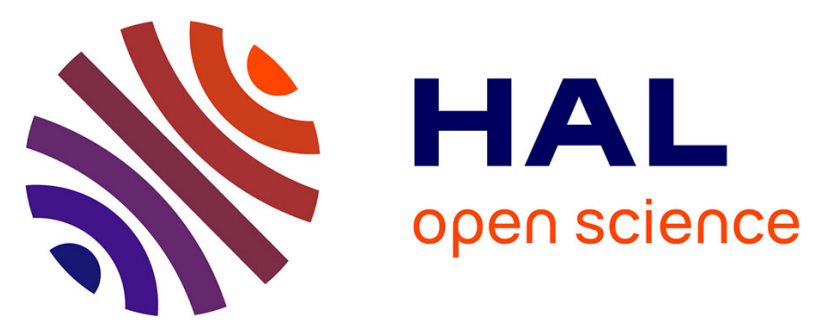

\title{
IV. TRANSPORTELECTRONIC STRUCTURE OF EUB6, TRANSPORT AND MAGNETIC PROPERTIES
}

\author{
T. Kasuya, K. Takegahara, M. Kasaya, Y. Isikawa, T. Fujita
}

\section{To cite this version:}

T. Kasuya, K. Takegahara, M. Kasaya, Y. Isikawa, T. Fujita. IV. TRANSPORTELECTRONIC STRUCTURE OF EUB6, TRANSPORT AND MAGNETIC PROPERTIES. Journal de Physique Colloques, 1980, 41 (C5), pp.C5-161-C5-170. 10.1051/jphyscol:1980528 . jpa-00219963

\section{HAL Id: jpa-00219963 https://hal.science/jpa-00219963}

Submitted on 1 Jan 1980

HAL is a multi-disciplinary open access archive for the deposit and dissemination of scientific research documents, whether they are published or not. The documents may come from teaching and research institutions in France or abroad, or from public or private research centers.
L'archive ouverte pluridisciplinaire HAL, est destinée au dépôt et à la diffusion de documents scientifiques de niveau recherche, publiés ou non, émanant des établissements d'enseignement et de recherche français ou étrangers, des laboratoires publics ou privés. 


\title{
IV. TRANSPORT.
}

\author{
ELECTRONIC STRUCTURE OF EUB 6 , TRANSPORT AND MAGNETIC PROPERTIES
}

T. Kasuya, K. Takegahara, M. Kasaya, Y. Isikawa and T. Fujita

Department of Physics, Tohoku University, Sendai, Japan.

\begin{abstract}
Rêsumé.- On montre que dans les composés non magnétiques $R^{++} B_{6}$, le défaut le plus courant, la lacune $\mathrm{R}^{++}$, peut piéger un troisième trou en créant un électron de conduction comparateur, ceci à cause du caractère de semiconducteur à bande interdite étroite. Cet electron est faiblement piégé et donc donne lieu facilement à une conduction de type $n$. Différentes propriétés de transport sont expliquées par ce modèle. Dand $E_{u B}$ magnétique le défaut précédent forme un amas à moment géant. De plus, à cause de l'effet d'anti-liaison avec les niveaux $4 f$, les tander de valence de spin parallèle sont faussées à 1 intérieur des tander de conäuction dans 1'état ferromagnétique, créant plusieurs électrons de conduction. Différentes propriétées anormales magnétiques et de transport sont expliquées dans ce modèle.
\end{abstract}

Ahstract.- In non-magnetic $\mathrm{R}^{+{ }^{+}} \mathrm{B}_{\sigma^{\prime}}$ it is shown that the most common defect $\mathrm{R}^{++}$ vacancy can trap third hole creating a compensating conduction electron due to the narrow gap semiconductor character. The latter is trapped loosely and thus easily causes n-type character. Various transport properties are explained on this model. In magnetic EuB, the above defect center makes a giant moment cluster. Furthermore, due to the antibonding effect with the $4 \mathrm{f}$ levels, the valence bands of the parallel spin are pushed up into the conduction bands in ferromagnetic state creating many conduction electrons. Various anomalous magnetic and magneto transport properties are explained on this model.

1. Introduction.- Rare earth hexaborides, $\mathrm{RB}_{6}$, have the CsCl type structure in which an octahedral $\mathrm{B}_{6}$ molecule replace a $\mathrm{Cl}$ atom. The bonding bands of the boron network require two extra electrons per cell and thus the divalent rare earth hexaborides are insulators. Both experiment and band calculation show that the gap between the valence and the conduction bands is very narrow, a few tenths of ev $/ 1,2,3 /$. The most probable defect of this structure is the $\mathrm{R}^{++}$vacancy /4/ which should, in usual sense, causes the p-type character. On the contrary, however, nearly all materials measured so far are n-type. At first, we discuss this peculiar problem as the character of the impurity state in narrow band semiconductors because the characteristic properties of this type of impurity state have also very important magnetic effects in $\mathrm{EuB}_{6}$.

EuB $_{6}$ had been reported to be a ferromagnet with a Curie temperature $T_{C}$ of about $10 \mathrm{~K} / 5 /$. The first single crystal made by us was, however, complicated antiferromagnetic type showing various kinds of anomalous properties /6/. Careful sample preparation and measurements on various physical properties indicate again that the purest $\mathrm{EuB}_{6}$ is ferromagnetic and the single crystal we made before includes a few percent of carbon $/ 7,8 /$. However, detailed investigation on pure $\mathrm{EuB}_{6}$ raised new puzzles that purest $\mathrm{EuB}_{6}$ is also very anomalous in its magnetic and electronic properties /9,10/. In $\$ 3$, we treat this problem on the stand point that the valence bands couple strongly with the $4 \mathrm{f}$ states causing very unusual behavior and, even in purest sample, the unusual impurity state mentioned before plays an important role. In \$4, anomalous properties of carbon doped $\mathrm{EuB}_{6}$ are treated on the same stand point.

2. Electronic Structure of $\mathrm{YbB}_{6}$.- As a typical non-magnetic divalent hexaboride, $\mathrm{YbB}_{6}$ is considered in detail. In figure 1 , electrical resistivities of pure and carbon doped $\mathrm{YbB}_{\sigma}$ are shown up to $1,000 \mathrm{~K}$ of temperature. It is apparent that in pure $\mathrm{YbB}_{6}$ the effect of the valence bands becomes clear above room temperature, while in carbon doped sample no effect is observed up to $800 \mathrm{~K}$ because of a large Fermi energy. 


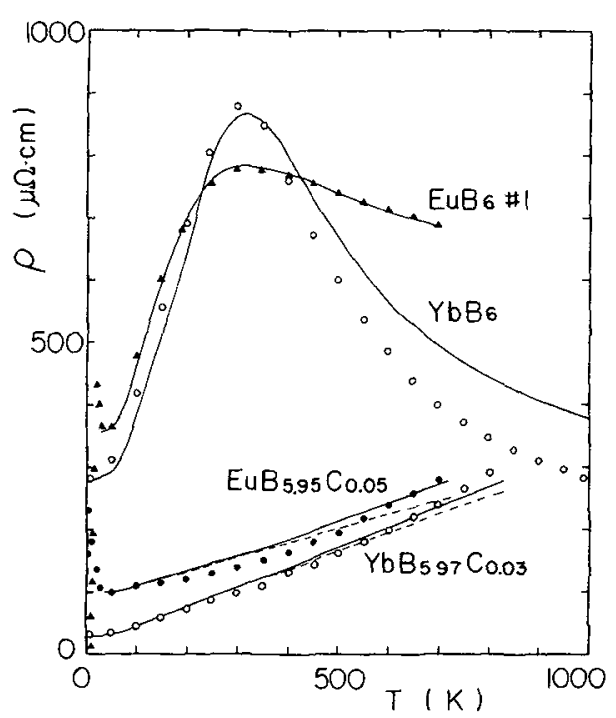

Fig. 1: Experimental values of the electrical resistivities are shown by o for $\mathrm{YbB} 6$ and $\mathrm{YbB}_{5} .9{ }^{\mathrm{C}} \mathrm{C} .03,4$ for $\mathrm{EuB}_{6} \mathrm{Al}_{1}$ and - for $\mathrm{EuB}_{5}{ }_{95} \mathrm{C} \cdot 95 / 24 /$. Calculated fitting curves are shown by solid Iines. See $T$ able I for the fitting parameters. For carbon doped samples, the calculated values for $\mathrm{B}=0$ are shown by broken lines.

From these facts, the top of the valence bands is expected to be about $1,000 \sim 2,000$ $\mathrm{K}$ below the bottom of the conduction bands. From the Hall constant, the number of the conduction electron $\mathrm{n}_{\mathrm{c}}$ at $300 \mathrm{~K}$ is $17 \%$ larger than that at $77 \mathrm{~K}$ in $\mathrm{YbB}_{6}$ but is nearly constant in carbon doped $\mathrm{YbB}_{6}$ as shown in table I.
Note that all of them are n-type. It is now well established both from experiments and from band calculations that both the bottoms of the conduction bands and the tops of the valence bands are at the three $x$ points. $T$ he effective mass of the conduction bands at the Fermi energy of $\mathrm{LaB}_{6}$ is about $0.5 \mathrm{~m}$, becoming smaller with decreasing energy, and is expected to be $0.2 \sim 0.3 \mathrm{~m}$ near at the bottom $/ 11,12,13 /$. On the other hand, the effective mass of the valence band is expected to be much larger at the top but there are no definite experimental evidences. Now let us consider at first the mechanism for the n-type character in most samples. The most probable defect is considered to be $\mathrm{R}^{++}$vacancy /4/ because the borons make a stable bonding network. Then, as was shown before by us $/ 14 /$, even the third extra hole can be localized mostly on the nearest neighbour $B_{6}$ molecules with a binding energy of about a few tenths of $\mathrm{eV}$ and then this impurity hole state is pushed up into the conduction bands and thus creates one compensating conduction electron. This electron is trapped loosely to the impurity complex with one positive effective charge but is very easily delocalized showing n-type conduction because of a small effective mass and the screening

Table Ia : Transport properties of carbon doped samples $\mathrm{RB}_{6} \mathrm{C}_{\mathrm{X}}$. The numbers of conduction electrons at $77 \mathrm{~K}$ and $300 \mathrm{~K}$ are fitted. Various fitting parameters shown in the text, $A, E_{O}, a, E_{i j}$ and $E_{i}$ are tabulated. $E_{F O}$, the Fermi energy at $\mathrm{T}=0, \rho_{4} .2$, resistivity at $4.2 \mathrm{~K}$, and the number of the $\mathrm{Eu}$ defect $\mathrm{n}_{i}$ are also shown.

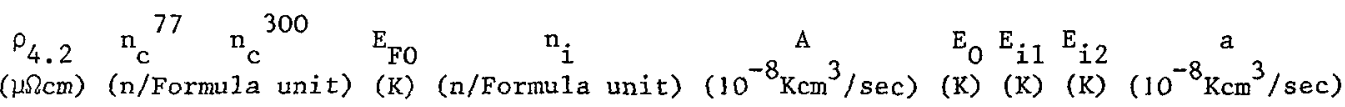

\begin{tabular}{llllllllll}
$\mathrm{YbB}_{5,97} \mathrm{C}_{0.03}$ & 31 & 0.021 & 0.021 & 3600 & 0.01 & 22.49 & 051002100 & 8.10 \\
\hline $\mathrm{EuB}_{5,95} \mathrm{C}_{0.05}$ & 170 & 0.023 & 0.023 & 3757 & 0.03 & 17.98 & 50052572257 & 27.59 \\
\hline
\end{tabular}

$\mathrm{EuB}_{5.97} \mathrm{C}_{0.03} \quad 0.03 \quad 0.03$


Table Ib : Transport properties of pure $\mathrm{RB}_{6} \cdot \mathrm{n}_{\mathrm{C}}{ }^{77}$ and $\mathrm{n}_{\mathrm{C}}{ }^{300}$ are fitted. Fitting parameters, $a, A, E_{O}, E_{i}$ and $\alpha$ are tubulated.

\begin{tabular}{|c|c|c|c|c|c|c|c|c|c|c|c|}
\hline & $\begin{array}{r}\rho_{4.2} \\
(\mu \Omega\end{array}$ & $p_{30}$ & $\begin{array}{c}\mathrm{n}_{\mathrm{c}}^{77} \\
(\mathrm{n} / \text { Formu }\end{array}$ & ${ }_{\mathrm{c}}^{300}$ & $\begin{array}{l}\mathrm{E}_{\mathrm{FO}} \\
(\mathrm{K})\end{array}$ & $\frac{n_{i}}{(n / \text { Formula unit) }}$ & $\left(10^{-8} \mathrm{Kcm}^{3} / \mathrm{sec}\right)$ & $\begin{array}{l}\mathrm{E}_{\mathrm{O}} \\
(\mathrm{K})\end{array}$ & $\begin{array}{r}E_{i} \\
(K)\end{array}$ & $\alpha$ & $\left(10^{-8} \mathrm{Kcm}^{3} / \mathrm{sec}\right)$ \\
\hline $\mathrm{YbB}_{6}$ & 280 & & 0.00056 & 0.00064 & 321 & 0.0008 & 10.12 & -1500 & 595 & 0.7 & 6.23 \\
\hline $\mathrm{EuB}_{6}^{\# 1}$ & 12 & 350 & 0.0015 & 0.0022 & 609 & 0.0017 & 24.73 & -180 & 1217 & 0.9 & 14.43 \\
\hline$\# 2$ & 35 & 800 & 0.0055 & 0.0067 & 1447 & 0.0079 & 24.73 & 900 & 1721 & 0.7 & 58.69 \\
\hline \#3 & 10 & 350 & $0.004(?)$ & & 1170 & 0.0015 & 24.73 & 0 & 1171 & 0.5 & 27.65 \\
\hline
\end{tabular}

effect. It is expected that the holes are localized even for a few percent of the vacancies, while the electrons are delocalized even for $0.01 \%$ of vacancies which are expected to be even in the purest sample. With increasing number of $\mathrm{R}^{++}$vacancies, the Fermi energy of the delocalized conduction electrons increases and the third hole band will be filled partly. This seems to be the situation of the so called pure $\mathrm{YbB}_{6}$. The situation is illustrated in figure 2 .

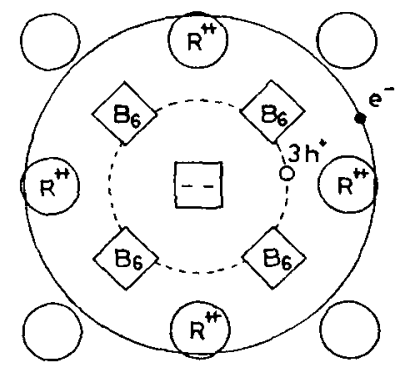

Fig. 2 : Schematic picture for a $\mathrm{R}^{++}$defect center.

When carbons are doped, each of which substitutes a boron and thus supplies one conduction electron, the thixd holes around $\mathrm{R}^{++}$vacancies are filled easily, and furthermore the second and finally the first holes are also filled. The situation of a few percent carbon doped sample seems to be in such a state and, for more than several percent of doped carbon, all the impurity holes seem to be filled causing normal magnetic and transport properties. Note that the hole impurity states are not the real localized states but the virtual bound state mixed with the conduction bands, in the similar sense as the usual d virtual state in the transition metal impurities. Note, however, that, because of the large correlation effect compared with the mixing energy, the nearly complete orbital splitting is expected in contrast with the $3 d$ case. The width of the virtual bound state is thought to be the same order of the energy of the conduction band at the bound state, that is, 1,000 2,000 K. The width due to the potential fluctuation is also expected to be the similar order.

Next, the resistivity of carbon doped sample is considered. When the scattering matrix is constant, the phonon scattering resistivity $\rho_{p}$ is inversely proportional to the Fermi energy $E_{F}$. Actually, the relation is applicable in comparison with ${ }_{\text {LaB }}{ }_{6}$, that is, $E_{F}=0.26$ and $2 \mathrm{eV}$ for the effective mass ratio $m_{c} / m=0.3$ and 0.5 , and $\rho_{\mathrm{p}}=70$ and $9 \mu \Omega \mathrm{Cm}$ for $\mathrm{YbB}_{5.97^{\mathrm{C}} .03}$ and $\mathrm{LaB}_{6}$ ' respectively. An unusual property in $\mathrm{YoB}$ $5.97^{\mathrm{C}} .03$ is the sharper than $T$ linear increase of the resistivity in high temperature region. This is attributed to the situation that the Fermi level is in between the second and the first bound states. Note that as the carbon concentration increases, the $\mathrm{Yb}$ vacancies also increase by compensation. In the present sample, 
about $1 \%$ of $\mathrm{Yb}$ vacancies are expected to be formed absorbing $1 \%$ of conduction electrons supplied by carbon leaving $n_{c}=0.02 / Y b$ as shown in table I. As an example, the calculated results based on the model shown in figure 3 are compared with experiment in figure 1. The electrical resistivity $\rho$ is calculated by the following form

$\rho^{-1}=e^{2} I_{00}$

$$
\begin{gathered}
L_{n m}=\frac{2 \nu\left(2 \pi{ }_{c}\right)^{1 / 2}}{3 \pi^{2} h^{3}} \int_{0}^{\infty} E^{3 / 2}(E-\mu)^{n+m} \cdot \tau(E) f(E) \\
\times(1-f(E)) \frac{d E}{k T}
\end{gathered}
$$

in which we assumed that only the conduction electrons carry the currents. Here, $v=3$ is the number of the valleys in the conduction bands, $\mu$ the Fermi energy, $f(E)$ the Fermi distribution function. The effective mass near the bottom of the conduction band is taken to be $m_{C}=0.25 \mathrm{~m}$. The life time $\tau(\mathrm{E})$ is given by

$$
\begin{aligned}
& \tau(E)^{-1}=a\left[N_{C}(E)+B\left(N_{i}(E)+N_{v}(E)\right)\right] \\
& +A \rho_{O P}(\hbar \omega / T)\left[N_{C}(E)+B\left(N_{i}(E \pm \hbar \omega)+N_{v}(E \pm \hbar \omega)\right)\right]
\end{aligned}
$$

in which the first term means the impurity scattering and the second term the phonon scattering with

$p_{\text {op }}(x)=x n(x)(1+n(x))$

in which $\mathrm{n}(\mathrm{x})$ is the Bose distribution function. Several comments should be done concerning the above form. (i) The density of states for the conduction band is given by the usual effective mass form of the three valleys. (ii) Concerning the valence bands, we have not enough information. A very interesting information comes from $\mathrm{KB}_{6}$, which should be metallic with a hole per $K$ atom but is an insulator with a small gap experimentally /15/. A possible interpretation may be as follows. The characteristic property of the top of the valence band is the non-bonding character in [100] directions /16/. Then, the Fermi surface of $\mathrm{KB}_{6}$ is expected to be formed by three nearly parallel pipes with a rather rectangular cross section due to the non-linear disper- sion in the perpendicular directions. Then we can expect formation of multiple charge density waves probably with six [110] directions of the wave vectors, or may be three [100] directions, which create a small gap. In this respect, the valence band is described rather well by the two dimentional dispersion. At the top of the valence band with a small number of the hole, however, three dimensional dispersion seems to be applicable, and we use the effective mass $m_{h}=2 m$ in the following calculation, which is larger than that of the band calculation $/ 3 /$ but agrees better with experimental results. (iii) The shape of the virtual bound state is assumed to be as follows,

$N_{i}(E)=n_{i} \frac{3}{4}\left(\frac{2}{\Delta}\right)^{3}\left[\left(\frac{\Delta}{2}\right)^{2}-\left(E-E_{i}\right)^{2}\right]$

which gives the number of the defect $n_{i}$ by integration with respect to $E$. The binding energy $E_{i}-E_{O}$, in which $E_{O}$ means the top of the valence bands, is expected to decrease with increasing number of $n_{C}$ (iv) Inelastic character should be included in the phonon scattering to the valence bands. This effect is, however, not important in the scattering to the conduction bands and then neglected.(v) The scattering to the virtual bound states should be treated in principle on the stand point of the resonance scattering. In the present calculations, however, we used the more conventional model and treated the valence band and the virtual bound state similarly. More careful check of this point will be done in the forthcoming paper. (vi) In usual ionic crystal, an electron near the bottom of the band is scattered mostly by the longitudinal optical phonons because the coupling constant is inversely proportional to the scattering vector $q$. With a finite number of conduction electrons, the coupling constant as well as the optical phonon frequencies is screened very quickly. This screening process depends sensitively on temperature because the numbers of conduction electrons and, more importantly, valence electrons change with temperature, 
which causes sensitive effect on the transport.

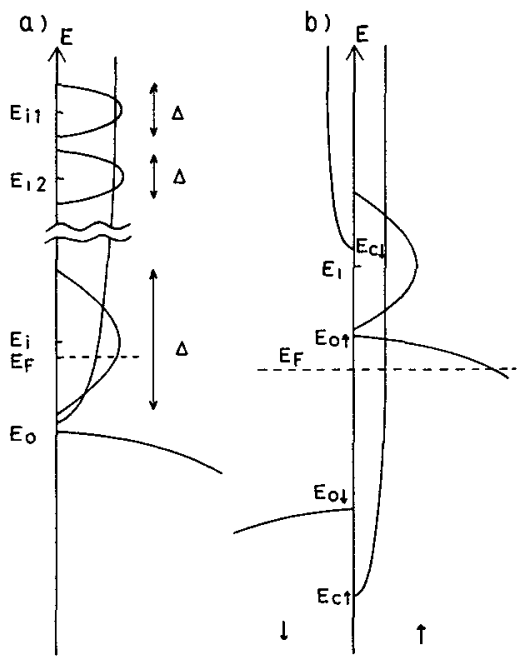

Fig. 3 : Density of states $N(E)$ is shown as a function of energy $E$ for a; paramagnetic region and $b$; ferromagnetic region for up and down spins.

We found, however, that various transport phenomena are not fitted consistently with the intravalley optical phonon scattering but are fitted well by the intervalley scattering. In this sense, the latter model was used with the typical phonon $\hbar \omega=$ $300 \mathrm{~K} / 17,18,19 /$. (vii) The parameter a is given so as to fit the residual resistivity. $B$ is chosen to be a constant 0.5 . Then, actual adjustable parameters are $A, E_{O}$ and $\alpha$, the fraction of unoccupied part of the bound state. These parameters are given in table I. In carbon doped samples, the energy difference between the second and the first bound states is chosen tentatively to be 3 , Oook.

In the case of $\mathrm{YbB}_{6}$, sharp decrease of the resistivity is observed above room temperature, which is due to the sharp increase of the conduction electron creating holes in the valence band, where $E_{O}$ is chosen to be $-1500 \mathrm{~K}$. Note that in the carbon doped sample the sharp increase of $n_{c}$ occurs above $1,000 K$. The value of $A$ is small in $\mathrm{YbB}_{6}$ compared to other samples. $T$ he reason is not clear. $\alpha$ is evaluated to be near unity, 0.8 to 0.9 , which suppresses $n_{C}$ at lower temperature and thus important to fit $n_{c} 300$.

3. $\underline{\mathrm{EuB}}_{6}{ }^{-}-$At first, let us consider the resistivity of a carbon doped sample EuB $_{6-x}$ $C_{x}, x=0.05$, in the paramagnetic region. Because the number of conduction electrons is similar to that of carbon doped $\mathrm{YbB}_{6}$ ' the behavior of $\rho$ is also similar. One remarkable feature is the stronger concave character, which is consistent with the larger number of the defect expected from the larger number of doped carbon. See the broken curves in figure 1, which neglect the impurity states. Another character is the larger residual resistivity, more than three times as large as that of $\mathrm{YbB}_{5.97^{C}} \mathrm{C}^{\circ}$ which is also consistent with the fact that the number of the defect is also expected to be about three times. Remaining fraction should be due to the d-f exchange scattering, which is estimated from another carbon doped sample, $\operatorname{EuB}_{5.97} \mathrm{C}_{0.03}$, to be about $25 \mu \Omega \mathrm{cm}$ as shown in figure 4 .

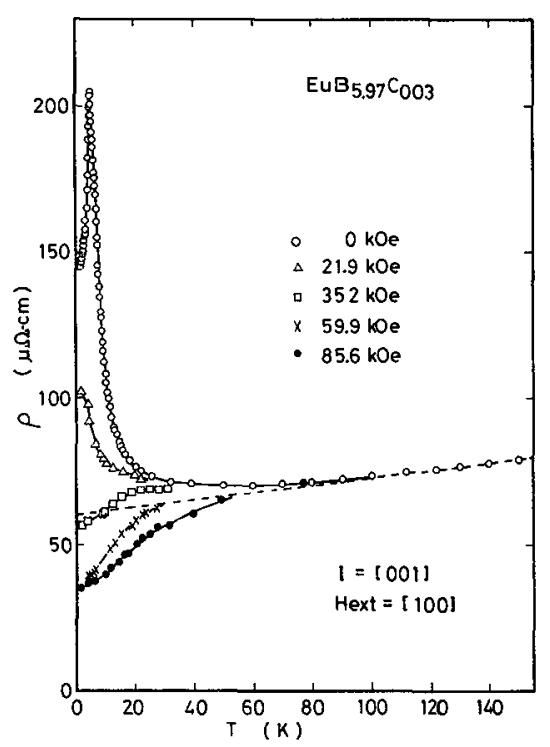
Fig. 4 : Resistivities of $\mathrm{EuB}_{5}, 9{ }^{\mathrm{C}}$ under
various magnetic fields shown ${ }^{3}$ the $\rho_{\text {mag }}$ is estimated from the dotted line and the value for $85 \mathrm{Koe}$.

From this value, we can estimate the square average value of the $d-f$ exchange 
constant $I_{\mathrm{df}}^{\text {eff }}=\left\langle\mathrm{I}_{\mathrm{df}}^{2}\right\rangle^{1 / 2}$ to be $0.035 \mathrm{eV}$ for $\mathrm{m}_{\mathrm{c}}=0.25 \mathrm{~m}$. While, in $\mathrm{GdB}_{6}, \mathrm{I}_{\mathrm{df}}^{\text {eff }}$ is estimated to be $0.05 \mathrm{eV}$ for $\rho_{\mathrm{mag}}=15 \mu \Omega \mathrm{cm} / 20 /$, which is consistent with the fact that the $5 d$ atomic character is about $50 \%$ at the bottom of the conduction band, increasing to $70 \%$ in the midale of the band $/ 3,13 /$.

In $\mathrm{EuB}_{6}$, the resistivity depends sensitively on sample. In figure 5, we show three examples, \#1, 2 and 3 . \# 1 is considered to be purest, \#2 includes about four times of defects and \#3, reported by Fisk et al./10/, may have the same amount of the Eu-vacancies as that of \# 1 and a larger amount of some n-type impurities, probably A. $/ 21 /$.

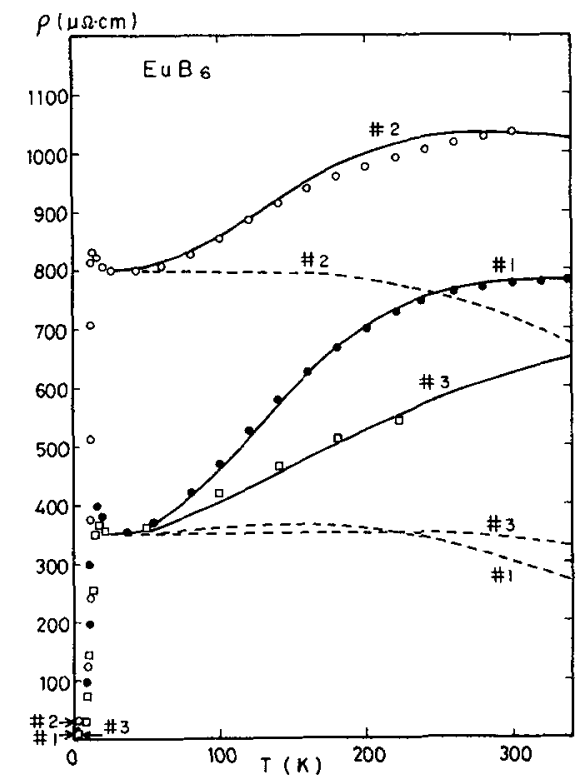

Fig. 5 : Resistivities of three typical examples of $\mathrm{EuB}_{6}$. Experimental values are shown by $\because$ and 0 for \#1, \#2 and \#3 samples, respectively. Calculated total resistivities are shown by solid lines and the disordered scattering term by broken curves. The values at $4.2 \mathrm{~K}$ are shown by arrows. -

Let us consider the paramagnetic region. The bahavior of \# 1 is similar to $\mathrm{YbB}_{6}$ but the relative change of $\mathrm{n}_{c}$ at room temperature is larger in \# 1 even if $\mathrm{n}_{\mathrm{C}} 77$ is larger. This means that the valence band is closer to, or may overlap with, the conduction band. one possible mechanism is the antibonding effect with the $4 \mathrm{f}$ states, which is expected to be about lev below the bottom of the conduction band /22/. Both the Hall effect and the resistivity are fitted well by using the parameter listed in table I. Note a nearly unity value of $\alpha$. Weaker decrease of resistivity above room temperature compared with $\mathrm{YbB}_{6}$ seen in figure 1 is simply due to a larger initial value of $\mathrm{n}_{\mathrm{c}}$. Note that, among the observed residual resistivity, the $d-f$ exchange resistivity is evaluated to be about $180 \mu \Omega \mathrm{cm}$, attributing remaining $170 \mu \Omega \mathrm{cm}$ to the defect scattering resistivity. Note also that the elastic scattering resistivity also shows fairly large temperature dependence as shown by broken line in figure 5 due to the temperature dependence of $\mu$ and $n_{c}$. In \# 2 sample, the residual resistivity is large because, compared with $n_{c^{\prime}}$, the number of defect $n_{i}$ is large and furthermore each impurity scattering is larger because of a small value of $\alpha$, indicating the near resonance scattering. Fitting parameters are shown in table 1. In \#3 sample, $n_{i}$ is expected to be similar to \# 1 and $n_{c}$ is supplied mostly by shallow n-type doners. In this case, too, a smaller value of a keeps the residual resistivity in the same level as \# 1 and lower the temperature dependence of the total resistivity. Note that the binding energy of the virtual bound state decreases with increasing number of conduction electrons, and, as the Eu-vacancies increase, the energy of the conduction band is lowered relatively to that of the valence band, which keeps the third bound state near the Fermi level causing relatively small decrease in $\alpha$. For the case of shallow donors, lowering of the conduction band seems to be weak.

With decreasing temperature, a giant moment is formed mostly by the six nearest neighbour Eu sites of each Eu-vacancy because a large indirect ferromagnetic exchange interaction exists through the vacant valence holes (bonding effect). The energy shift of the valence band (4f level) 
due to the antibonding (bonding) effect is evaluated by the band calculation to be about 2,000K for the full polarization of the $4 \mathrm{f}$ spin $\mathrm{S}$, which is consistent with a rough estimation of the atomic picture. This gives $I_{v}{ }^{2} 300 \mathrm{~K}$ in the form of $I_{v} S \alpha_{v^{\prime}}$ in which $\alpha_{v}$ is the Pauli operator of the valence electron. Therefore, the giant moment around a defect is estimated to be formed at about $50 \mathrm{~K}$. Once the giant moment is formed, the energy of the bound hole is pushed up causing increase in the Fermi energy and thus $n_{c}$. In the ferromagnetic region, the valence band of up spin shifts up about $1,000 \mathrm{~K}$ and down spin down 1,000K. On the other hand, the conduction bands move oppositely. The intraband $d-f$ exchange constant $I_{C}$ is evaluated to be about $600 \mathrm{~K}$ both from $I_{\text {eff }}$ estimated before and assuming that the intervalley interaction is small and the $50 \%$ of the $5 \mathrm{~d}$ character at the bottom of the conduction band and the atomic d-f exchange $I_{d f}^{O}=0.1 e V . T$ hen the shifts of the conduction bands are about $¥ 2000 \mathrm{~K}$ for \pm spins. The situation is illustrated in figure $3 b$. A remarkable result is large increase of $n_{c}$. Actually, in various ${ }^{E u B}{ }_{6}, n_{c}$ obtained from the Hall constant at $4.2 \mathrm{~K}$ increases five \pm one times compared with that at $77 \mathrm{~K}$. The following combinations of $I_{c}$ and $I_{v}$ are consistent with the above result; $\left(I_{c}, I_{V}\right)=(465,465)$ and $(620,310)$ $\mathrm{K}$. As was estimated before, the real combination seems to be closer to the latter. Decrease of the resistivity is larger than $\mathfrak{n}_{c^{\prime}}$ in \#1, for example, $1 / 35$ for the total residual resistivity and $1 / 17$ for the defect scattering resistivity. This means that the defect scattering constant decreases rapidly with increasing number of $\mathrm{n}_{c}$. For example, in \# 2, the residual resistivity ratio is $1 / 23$ and in the ferromagnetic region $n_{c}=0.028 / \mathrm{Eu}$ and $\rho_{O}=35$

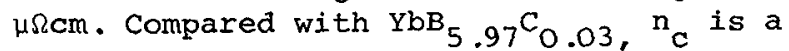
little bit larger and the defect concentration is a little bit smaller but $\rho_{O}$ is nearly equal. This difference is explained consistently by the coexistance of the valence band holes in \#2.

The magnetic properties may be evaluated in the following ways. Here, only the free part, which means outside of the giant moment, is treated and the effest of the giant moment is treated by the effective field $\mathrm{H}$ or the effective moment $\mathrm{M}_{\mathrm{O}}$. (More detailed treatment including the giant moment self-consistently will be given in a separate paper.)

$\mathrm{E}_{C \sigma}=E_{C}^{O}-I_{C} \sigma\left(M_{f}+\alpha M_{O}\right)-\mu H \sigma$

$E_{v \sigma}=E_{v}^{O}+I_{v} \sigma\left(M_{f}+\beta M_{O}\right)-\mu H \sigma$

$F=F_{e}-g \mu H M_{f}-J M_{f}^{2}-k T \phi\left(M_{f}\right)$

in which $M_{f}=\left\langle S_{f}\right\rangle, F_{e}$ is the Free energy of the conduction and the valence electrons per $E u$ atom and $\phi(M)$ is the entropy of each $4 \mathrm{f}$ spin. $M_{f}$ is determined by minimizing $F\left(M_{f}\right)$. In figures 6 and 7 , temperature dependence of $M$ and $C$, specific heat, is shown. In the case $I_{,} I_{C}=I_{V^{\prime}}$, the transition occurs at $\mathrm{T}_{\mathrm{C} 2}=7 \mathrm{~K}$ in the first order promoted by the valence band. In the case II, $I_{C}=2 I_{V}$, the usual second order transition occurs at about $T_{C 1}=3 \mathrm{~K}$ due to the conduction electrons. When the direct ferromagnetic coupling between $4 \mathrm{f}$ spins is introduced, which is expected to be the same mechanism as that in Eu-chalcogenides treated by us /23/ and the value $\mathrm{J}=0.4 \mathrm{~K}$ is also reasonable, $\mathrm{T}_{c_{2}}$ remains nearly constant but $\mathrm{T}_{\mathrm{cl}}$ is enhanced and increases to $13 \mathrm{~K}$ in agreement with experiment. Below $\mathrm{T}_{\mathrm{cl}}$, however, saturation of the polarization of the conduction electron spin occurs easily and thus $M_{f}$ is kept small and the specific heat is also small. When the effect of the giant moment is included, the sharp transitions are smeared out and the agreement with experiment becomes better. Note that the result of our specific heat measurement agrees very well with that of Fisk et al./10/ below $12 \mathrm{~K}$, but above $12 \mathrm{~K}$ our value is about a half of theirs. This may be due to the difference of the defect number. Actually there is a long tail due to the giant moment formation (fig. 7).. 


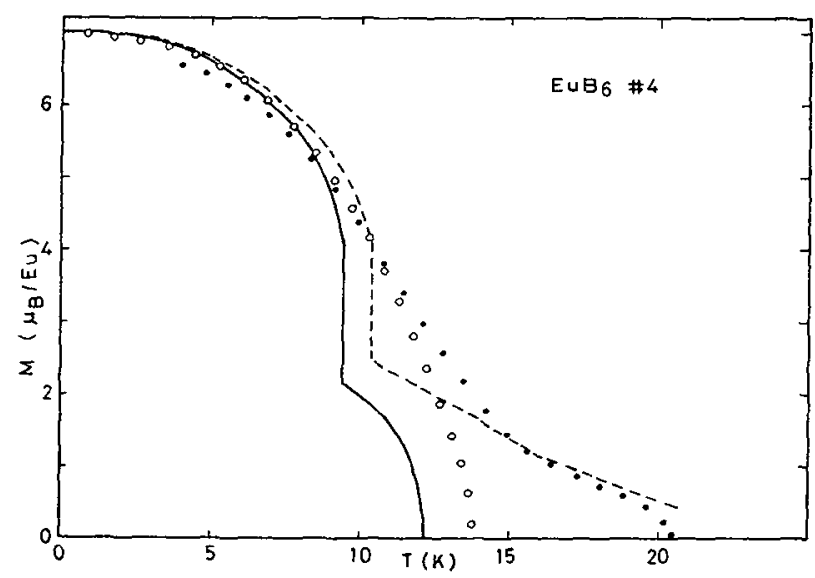

Fig. 6 : Magnetization $M$ of \# 4 sample are plotted as functions of temperature $T$. 0 is obtained from the extrapolation of the Arrott plot at $\mathrm{H}_{i \mathrm{j}} / \mathrm{M}=2 \mathrm{kOe} / \mu_{\mathrm{B}} / \mathrm{Eu}$ and $\cdot$ is the value at $\mathrm{H}_{\mathrm{in}}$ in $=0.5 \mathrm{kOe} / \mu_{\mathrm{B}}^{\mathrm{B}} / \mathrm{Eu}$. Solid curve is obtained by calculation for $I=$ $620 \mathrm{~K}, I=310 \mathrm{~K}, \mathrm{~J}=0.4 \mathrm{~K}$ and $\mathrm{M}=\mathrm{H}=0$. Broken curve is obtained by'including $\mu_{\mathrm{B}} \mathrm{H}=0.25 \mathrm{~K}$.

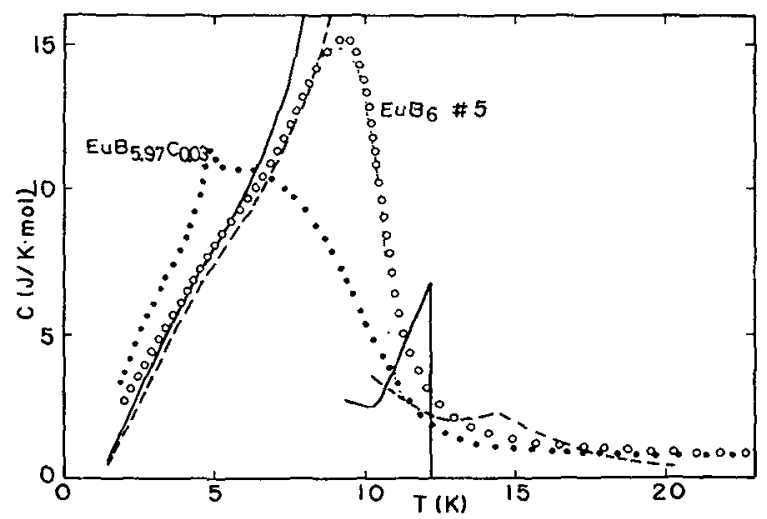

Fig. 7 : Magnetic specific heat $C$, obtained by subtracting that of $\mathrm{LaB}_{6}$, of \#5 EuB 6 ' for comparison, that of EuB ${ }_{97} \mathrm{C}$ are shown by ond ', respectivery? calculated values are shown' by solid and broken lines. The parameters used are the same as those in figure 6 . The dotted curve is the expected theoretical curve by taking into account a local fluctuation of the giant moment.

Note also that our molecular field model calculation fits the experimental $T$ linear dependence below $T_{\mathrm{c} 2}$ very well. From the Arrott plot, we found that $\mathrm{T}_{\mathrm{Cl}}$ is nearly constant, 13 $14 \mathrm{~K}$, in various samples which show different transport behaviors. This means that $\mathrm{T}_{\mathrm{Cl}}$ is intrinsic and the main effect of the giant moment around the defect is to smear the sharp transitions at $T_{C l}$ and $T_{C 2}$.

Finally, the transient temperature region near above $\mathrm{T}_{\mathrm{Cl}}$ is considered. There are two main effects. One is the giant moment formation as was described before. Another is the shift of the conduction and valence bands due to the short range order of $4 \mathrm{f}$ spins. The situation is illustrated schematically in figure 8 . There are variou anomalies both in transport and magnetic behaviors. For example, a peak is observed in resistivity several degrees above ${ }_{\mathrm{Cl}}$.

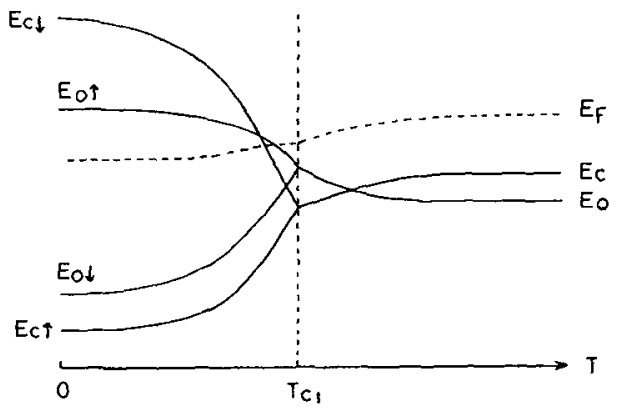

Fig. 8 : Schematic picture for the short range order effect. Top of the valence band $E_{O}$, bottom of the conduction band $E_{C}$ and Fermi energy $E_{F}$ are shown.

In figure 9 we show a most typical example of \# 4 polycrystalline sample, which shows the largest peak.

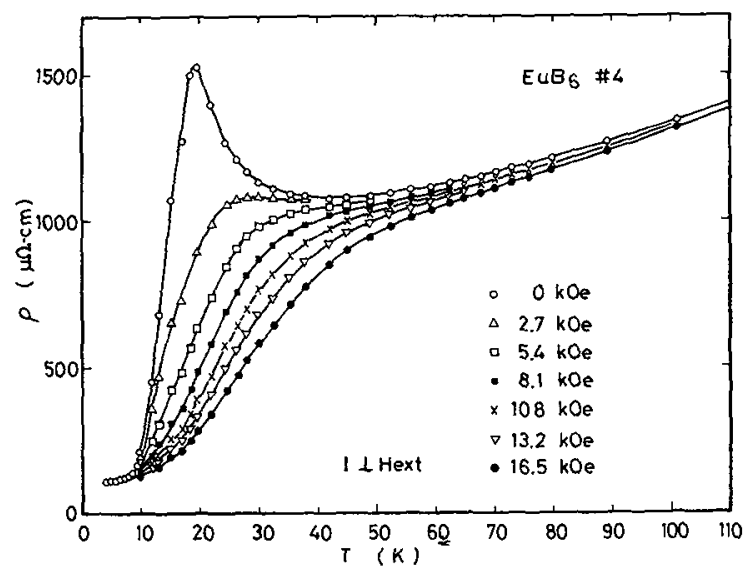

Fig. 9 : Electrical resistivity of polycrystaliine sample $\mathrm{EuB}_{6} \# 4$ under magnetic fields. 
Note that our polycrystalline samples give correct intrinsic informations on magnetic, electronic and transport properties such as Hall effect and thermoelectric power except the absolute value of resistivity. Various properties of \# 4 are very similar to these of \# 1 except a large peak of resistivity. $\mathrm{T}_{c}$ is evaluated to be $13.9 \mathrm{~K}$ from the detailed Arrot plot. As shown in figure 9, the peak disappears for only $2.7 \mathrm{kOe}$ of the applied field. Note that $\mathrm{n}_{\mathrm{C}}=0.0026 / \mathrm{Eu}$ at $300 \mathrm{~K}, 0.0016 / \mathrm{Eu}$ at $77 \mathrm{~K}$ and $0.008 \mathrm{l} / \mathrm{Eu}$ at 4.2K. Three possible mechanisms, resonance scattering by the giant moment, usual ferromagnetic critical scattering and shift of the top of valence band to the Fermi level by the short range ordering, are considered but it is difficult now to select one from others as the most important. A sharp structure is observed also in the thermoelectric power of $\# 4$ as shown in figure 10 .

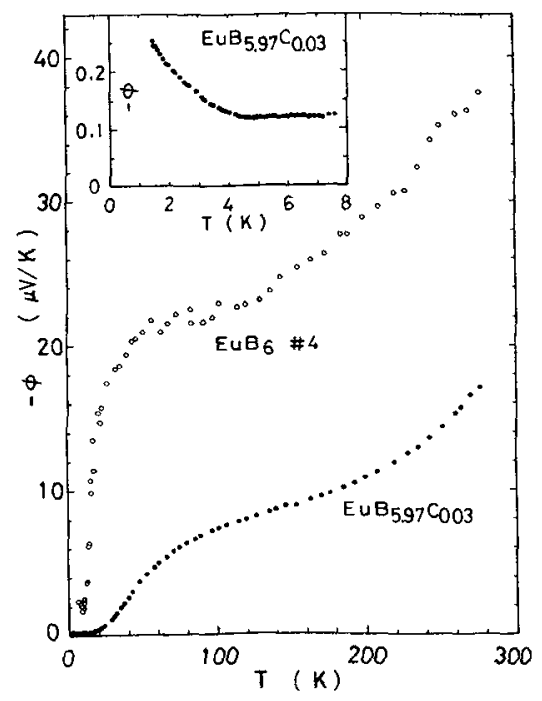

Fig. I0 : Thermoelectric power $\phi$ of $\mathrm{EuB}_{6}$ \# 4 is plotted as a function of $T$. For comparison, $\phi$ of $E^{E^{3}} .97^{C} .03$ is also shown.

For the thermoelectric power, more detailed informations on the energy dependence of the scattering matrixes are involved and we have not yet done the detailed quantitative fitting. But the sharp decrease at about $30 \mathrm{~K}$ seems to suggest the effect of giant moment formation. The giant moment effectis also seen in the induced moment above $T_{\mathrm{cl}}$ as shown in figure 6 .

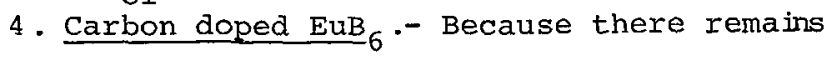
no space any more, this topic is sketched very briefly. For samples of $n_{c} \geq 0.02 n 0.03 /$ $\mathrm{Eu}$ in the paramagnetic region, the top of the valence band can not reach the Fermi level even in the perfect $4 f$ spin alignment and thus $n_{c}$ is nearly constant in the whole temperature region. Anomalous behavior is, thus, mostly due to the giant moment through the first hole trapped by the Eu defect. Furthermore, as $\mathrm{k}_{\mathrm{F}}$ increases, the intervalley exchange becomes important and thus the complicated spin ordering is rather realized. The detail will be given in a separate paper.

5 Conclusion.- Due to the narrow gap character, a $\mathrm{K}^{++}$vacancy can trap the third hole on the nearest neighbour $B_{6}$ molecules, which creates a compensating loosely trapped conduction electron. Various anomalous transport properties are explained by this model. At low temperature, the above defect center forms a giant moment in $\mathrm{EuB}_{6}$ and furthermore, the valence bands with the parallel spin are shifted up through the antibonding effect with the 4 f spins, creating a lot of conduction electrons and causing the first order transition to the strong ferromagnetism at $\mathrm{T}_{\mathrm{C} 2}$. The second order transition at a higher temperature $\mathrm{T}_{\mathrm{cl}}$ is due to the conduction bands and the direct $4 \mathrm{f}$ exchange interaction, which cause a weak ferromagnetism because the conduction electron spins saturate very quickly. The giant moments smear the sharp transitions at $\mathrm{T}_{\mathrm{Cl}}$ and $\mathrm{T}_{\mathrm{C}_{2}}$. Anomalous magnetic and transport properties in $\mathrm{EuB}_{6}$ are explained on the above model.

Some of the experimental data were measured in the Bordeaux University during one of the author (M.K.) had stayed there He expresses his cordial thanks to Professor P. Hagenmuller, Professor J . Etourneau and the staff members in Professor Hagenmuller's group for their kind hospita- 
lities. Comments and informations of Dr. A. Hasegawa on his band calculations are greatly appreciated.

\section{References}

/1/ Johnson, R.W. and Daane, A.H., J . Chem. Phys. 38 (1963) 425 .

12) Mercurio, J.P., Etourneau, J., Naslain, R., Hagenmuller, P. and Goodenough, J.B., J. Solid State Chem. 9 (1974) 37 .

13/ Hasegawa, $A$. and Yanase, A. J.Phys. C, 12 (1979) 5431 .

/4/ Etourneau, J., Naslain, R. and La Placa, S., J. Less Common Met, 24 (1971) 183 .

15/ Matthias, B.T.., Geballe, T.H. Andres, K., Corenzwit, E., Hull, G.W. and Maita, J.P., Science 159 (1968) 530.

/6/ Isikawa, Y., Bajaj, M.M., Kasaya, M. T anaka, T . and Bannai, E., Solid State Commun 22 (1977) 573. Also see Kasaya, M. and Isikawa, Y ., Extended Abstract of US J apan Seminar in Sendai, (1977) 171 and T. Kasuya ibid 183.

17/ Kasaya, M., T arascon, J .M., Etourneau, J. and Hagenmuller, P., Mater Res. Bull 13 (1978) 751 .

Kasaya, M., Tarascon, J.M., Etourneau, $J$., Hagenmuller, P. and Coey, J.M, J . Physique Colloq. 40 (1979) C5-393.

/8/ Coey, J .M.D., Massenet, O., Kasaya, $M$. and Etourneau, J ., J . Physique Colloq. 40 (1979) C 2-333.

19/ Isikawa, Y ., Thesis for Dr. of Science Tohoku University, 1977.

/10/ Fisk, Z ., Johnston, D.C, Cornut, B., Von Molnar, S., Oseroff, $S$. and Calvo R., J . Appl. Phys . 50 (1979) 1911.

/11/ Ishizawa, Y., Tanaka, T., Bannai, E. and Kawai, S., J . Phys. Soc. J apan 42 (1977) 112 .

12) Arko, A J : Crabtree, G., Karim, D. and Fisk, Z ., Phys. Rev. Bl3 (1977) 5240 .

/13/ Hasegawa, A. and Yanase, A., J . Phys. F., 7 (1977) 1245 .

/14/ Kasuya, T., J . Physique Colloq. 37 (1976) C4-261.

/15/ Naslain, R., Etourneau, J . and Hagenmuller, P., Boron and Refractory Borides (1977) 262 .

/16/ Longuet-Higgins, H.C. and De V. Roberts, M., Proc. Roy. SOC. 224A (1954) 336 .

Yamazaki, M., J . Phys. Soc. Japan, 12 (1957) 1 .
17/ Tanaka, T., Akahane, T., Bannai, E., Kawai, S., T suda, N. and Ishizawa, Y., J . Phys. C., 9 (1976) 1235 .

/18/ Etourneau, J ., Mercurio, J ., Naslain, $R$. and Hagenmuller, P., J . Solid State chem. 2 (1970) 332 .

/19/ Kasuya, T., Takagahara, K., Fujita, T., Tanaka, T. and Bannai, E., J . Physique Colloq. 40 (1979) C5-308.

/20/ Coles, B.R., Cole, T., Lambe, J . and Laurance, N., Proc. Phys. Soc. 79 (1962) 84 .

/21/ Private Communication.

/22/ Takakuwa, Y., Suzuki, S. and Sagawa. T... J apan. J . Appl. Phys . 17 (Suppl. 17-2) (1978) 284 .

/23/ Kasuya, T., IBM J . Res. Dev. 14 (1970) 214 .

/24/ The detail of the experimental results will be published in, Tarascon, J .M.; Etourneau, J . Dordor, P., Hagenmuller, P., Kasaya, M., and Coey, J.M.D., J. Appl. Phys. 